\title{
Jan Chrastina
}

From elementary algebra to Bäcklund transformations

Czechoslovak Mathematical Journal, Vol. 40 (1990), No. 2, 239-257

Persistent URL: http://dml.cz/dmlcz/102378

\section{Terms of use:}

(C) Institute of Mathematics AS CR, 1990

Institute of Mathematics of the Czech Academy of Sciences provides access to digitized documents strictly for personal use. Each copy of any part of this document must contain these Terms of use.

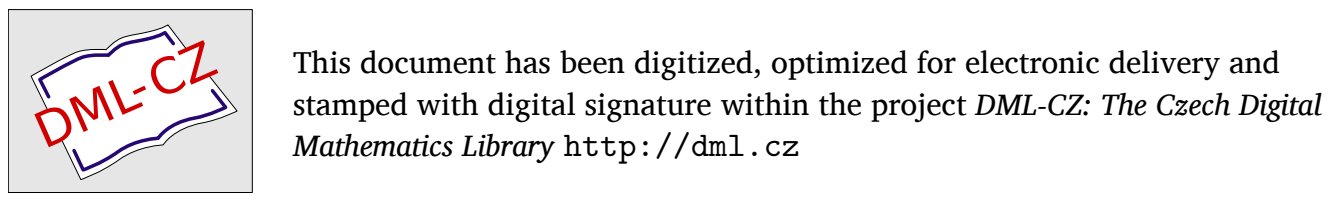




\title{
FROM ELEMENTARY ALGEBRA TO BÄCKLUND TRANSFORMATIONS
}

\author{
Jan Chrastina, Brno
}

(Received August 21, 1988; completed September 13, 1989)

It has been known for a long time that several areas of the theory of differential equations (compatibility theory, generalized symmetries, geometry of characteristics, variational complexes, and others) can be clarified by using certain infinite-dimensional spaces and algebraic tools that cannot be easily related with today's mathematical theory. The spectrum of these topics is rather wide and goes from linear algebra (infinite systems of linear equations) through vector fields in $\mathbb{R}^{\infty}$ (a generalization of Lie's theory) up to the axiomatic foundations of differential equations (naturally including the concept of Bäcklund correspondences). In dealing with all these topics, our exposition cannot be too punctilious and thorough for obvious reasons, many important problems must be left unsolved, and several interesting questions are tacitly passed over.

1. The Gauss elimination. We shall deal with a system $A$ and the relevant homogeneous system $\boldsymbol{A}$ of linear algebraic equations

$$
\begin{aligned}
& A^{i}: \sum a_{j}^{i} x^{j}=y^{i}, \quad A^{i}: \sum a_{j}^{i} z^{j}=0 \\
& (i=1,2, \ldots ; \text { sum over } j=1, \ldots, m(i))
\end{aligned}
$$

with real coefficients $a_{j}^{i} \in \mathbb{R}$ and three series of independent variables $x^{i}, y^{i}, z^{i}$. (The finite sum will be always indicated by $\sum$, while $\sum^{\infty}$ will be used for the infinite series.) We begin with strong assumptions: let $\boldsymbol{A}$ consist of linearly independent equations and admit only trivial solutions $z^{i} \equiv 0$. Under these assumptions, the systems $A, A$ will be modified by certain invertible rearrangements (permutation of equations, substitutions) but the intermediate results will be not always explicitly indicated by a change of notation.

With this in mind, we may ensure the nonvanishing of all left corner diagonal minors, det $\left(a_{j}^{i}\right) \neq 0(i, j=1, \ldots, n)$ for $n=1,2, \ldots$, permuting (if necessary) the equations. Then the "direct run" of the elimination can be applied. The equation $A^{1}$ is retained but $x^{1}$ calculated from $A^{1}$ and inserted into $A^{2}, A^{3}, \ldots$ ensure $a_{1}^{i}=0$ $\left(i>1\right.$ ). Then the second (new) equation $A^{2}$ is retained but $x^{2}$ calculated from $A^{2}$ and inserted into $A^{3}, A^{4}, \ldots$ ensures $a_{2}^{i}=0(i>2)$. Continuing with $x^{3}, x^{4}, \ldots$, 
the resulting system denoted by $B$ and the relevant homogeneous $\boldsymbol{B}$ are upper triangular with nonvanishing diagonal terms:

$$
\begin{aligned}
& B^{i}: \sum b_{j}^{i} y^{j}=\left\{y^{1}, \ldots, y^{i-1}\right\}+y^{i}, \quad B^{i}: \sum b_{j}^{i} z^{j}=0 \\
& \left(b_{j}^{i}=0 \text { if } i>j\right) .
\end{aligned}
$$

We denote by $\{\ldots\}$ a finite linear combination of the entries involved.

The "inverse run" is less trivial. Choosing a fixed $i$, the variables $x^{i+1}, \ldots, x^{i+c}$ calculated from $B^{i+1}, \ldots, B^{i+c}$ and inserted into $B^{i}$ yield a result like this:

$$
b_{i}^{i} x^{i}+r^{c}=\left\{y^{1}, \ldots, y^{i+c}\right\} \quad\left(r^{c}=\left\{x^{i+c+1}, x^{i+c+2}, \ldots\right\}\right) .
$$

In principle there are two possibilities: either $r^{c}=0$ for $c$ big enough (and $x^{i}$ is resolved) or $r^{c} \neq 0$ survive for all $c$. But in reality, the latter case cannot occur since it implies the existence of a nontrivial solution of $\boldsymbol{B}$, hence of $\boldsymbol{A}$, which is rejected. (In fact, $r^{c} \neq 0$ for all $c$ means that the form $\boldsymbol{B}^{i}-b_{i}^{i} z^{i}=b_{i+1}^{i} z^{i+1}+\ldots+b_{m(i)}^{i} z^{m(i)}$ is not a linear combination of $\boldsymbol{B}^{i+1}, \boldsymbol{B}^{i+2}, \ldots$ and one can then see that there exists a solution of $\boldsymbol{B}$ with $z^{1}=\ldots=z^{c}=0$ but with some nonvanishing $z^{c+1}, z^{c+2}, \ldots$, which is impossible.) Successively taking $i=1,2, \ldots$ we conclude that our algorithm when applied to $B$ leads to a diagonal system

$$
C^{i}: x^{i}=\sum c_{j}^{i} y^{j} \quad(i=1,2, \ldots ; \text { sum over } j=1, \ldots, n(i))
$$

of $B$, hence of the original system $A$.

2. Summary. If $\boldsymbol{A}$ consists of linearly independent equations and permits only trivial solutions, then $A$ is uniquely solvable by certain formulae $C$. Then the relevant homogeneous system $C$ satisfies the same conditions as $\boldsymbol{A}$ so that the relation between $A$ and $C$ is symmetrical. Moreover, if all left corner minors of $\boldsymbol{A}$ are nonvanishing, then the following criterion of solvability holds: for every $c=1,2, \ldots$ there exists $k(c) \geqq c$ such that the submatrix $\left(a_{j}^{i}\right)$ with rows $i=1, \ldots, k(c)$ and columns $j=c, c+1, \ldots$ is of rank $k(c)-c$. (All assertions easily follow from the above procedure of elimination.)

3. Bases and duality. We pass to a more conceptual point of view. Let $\boldsymbol{V}$ be an $\mathbb{R}$-linear space, $\boldsymbol{V}^{\wedge}$ the dual space of all $\mathbb{R}$-linear functions on $\boldsymbol{V}$. In view of future applications, the values will be written as $\xi(X) \in \mathbb{R}$ where $\xi \in \boldsymbol{V}, X \in \boldsymbol{V}^{\wedge}$. A sequence $\xi^{1}, \xi^{2}, \ldots \in \boldsymbol{V}$ is called a basis of $\boldsymbol{V}$ if every $\xi \in \boldsymbol{V}$ can be uniquely expressed as $\xi=$ $=\sum x_{i} \xi^{i}\left(x_{i} \in \mathbb{R}\right)$. One can see that such a sequence is a basis if and only if it is linearly independent and $\xi^{i}(X)=0$ implies $X=0$. If $\eta^{1}, \eta^{2}, \ldots$ with $\eta^{i}=\sum a_{j}^{i} \xi^{j}$ is an other basis, then there exists an inversion $\xi^{i}=\sum c_{j}^{i} \eta^{j}$. This provides a simpler but less constructive approach to the problem of Section 1 . A sequence $X_{1}, X_{2}, \ldots \in \boldsymbol{V}^{\wedge}$ is called a weak basis of $\boldsymbol{V}^{\wedge}$ if $\xi\left(X_{i}\right)=0$ for every $\xi \in \boldsymbol{V}$ and $i=i(\xi)$ big enough (therefore every series $\sum^{\infty} x^{i} X_{i}\left(x^{i} \in \mathbb{R}\right)$ makes a good sense), and every $X \in V$ can be uniquely expressed as $X=\sum^{\infty} x^{i} X_{i}$ with appropriate $x^{i} \in \mathbb{R}$. 
For every basis $\xi^{1}, \xi^{2}, \ldots$ of $V$ there exists a dual weak basis $X_{1}, X_{2}, \ldots$ determined by $\xi^{i}\left(X_{j}\right) \equiv \delta_{j}^{i}$. Moreover, every weak basis is of this kind. (In fact, let $Y_{1}, Y_{2}, \ldots$ be another weak basis of $\boldsymbol{V}^{\wedge}$. Then $Y_{j}=\sum^{\infty} a_{j}^{i} X_{i}$ where $a_{j}^{i}=\xi^{i}\left(Y_{j}\right)$ so that $a_{j}^{i}=0$ $(j>m(i))$. Conversely, $X_{j}=\sum^{\infty} e_{j}^{i} Y_{i}$ with

$$
X_{j}=\sum^{\infty} e_{j}^{i}\left(\sum^{\infty} a_{i}^{k} X_{k}\right)=\sum^{\infty}\left(\sum e_{j}^{i} a_{i}^{k}\right) X_{k} \quad \text { (a finite sum!). }
$$

Consequently $\sum e_{j}^{i} a_{i}^{k} \equiv \delta_{j}^{k}$. One can see that the relevant homogeneous system $\boldsymbol{A}$ satisfies the assumptions of Section 1 which implies $e_{j}^{i} \equiv c_{j}^{i}$, hence $e_{j}^{i}=0$ for $j$ big enough. So we may put $\eta^{i}=\sum e_{j}^{i} \xi^{j}$ and verify the duality $\eta^{i}\left(Y_{j}\right) \equiv \delta_{j}^{i}$ by direct calculations.)

4. The underlying spaces. We turn to the analysis. Let $\mathbb{R}^{\infty}$ be the space of all sequences $t=\left(t^{1}, t^{2}, \ldots\right)$ of real numbers equipped with the usual direct product topology. An infinite product of open intervals $a^{i}<t^{i}<b^{i}$ is called a box, a topological subspace $U \subset \mathbb{R}^{\infty}$ which is a union of such boxes is called an underlying space. Our reasoning will be carried out in such an underlying space $\boldsymbol{U}$ which will not be exactly specified and even fixed (to simplify the exposition).

Let $\mathscr{F}=\mathscr{F}(\boldsymbol{U})$ be the structural ring consisting of all $C^{\infty}$-smooth real valued functions $f=f\left(t^{1}, \ldots, t^{n(f)}\right)$ on $\boldsymbol{U}$ depending on a finite number of coordinates. Then the natural transformations $F$ are given by certain formulae

$$
F(t)=\bar{t}=\left(\bar{t}^{1}, \bar{t}^{2}, \ldots\right) \in \mathbb{R}^{\infty}, \quad \bar{t}^{i}=f^{i}\left(t^{1}, \ldots, t^{m(i)}\right) \in \mathscr{F} .
$$

If we are interested in the inversion

$$
F^{-1}(\bar{t})=t=\left(t^{1}, t^{2}, \ldots\right) \in \mathbb{R}^{\infty}, \quad t^{i}=g^{i}\left(\bar{t}^{1}, \ldots, \bar{t}^{n(i)}\right) \in \mathscr{F},
$$

the criterion of Section 2 can be applied to the differential $\mathrm{d} F$ and the presumed inversion $\mathrm{d} F^{-1}=(\mathrm{d} F)^{-1}$. With $a_{j}^{i} \equiv \partial f^{i} / \partial t^{j}, x^{j} \equiv \mathrm{d} t^{j}, y^{i} \equiv \mathrm{d} t^{i}$, this criterion leads to very strong requirements for the Jacobian matrix $\left(\partial f^{i} / \partial t^{j}\right)$, hence for the functions $f^{i}$. According to the usual finite-dimensional implicit function theorem, this criterion ensures that the part $t^{i}=g^{i}(i=1, \ldots, c)$ of the inverse formulae can be derived from the finite family $t^{i}=f^{i}(i=1, \ldots, k(c))$ of the original equations by elimination of the parasite variables $t^{c+1}, t^{c+2}, \ldots$ via a nonlinear version of the Gauss elimination of Section 1. Since the elimination proceeds only locally, the existence of all functions $g^{i}$ leads to the inverse mapping $F^{-1}$ only if the intersection $\cap U^{i}$ of the definition domains $\boldsymbol{U}^{i}$ of $g^{i}$ is wide enough, e.g., if it contains a model space. (A quite different but a little peculiar approach to this trouble will be suggested in the next section. Note aside that the requirement of inversion on an open subset of $\mathbb{R}^{\infty}$ is very restrictive for the common practise. For this reason, $\mathbb{R}^{\infty}$ cannot be considered an "infinite dimensional manifold" in the classical sense. Expressively we can say that manifolds are like the Banach spaces but $\mathbb{R}^{\infty}$ behaves as the linear topological space; the boxes may be compared with bounded subsets, not with open balls.) 
5. One-parameter groups. To ensure nontrivial results, a somewhat peculiar measures are needed. Let $F_{\lambda}$ be introduced by

$$
F_{\lambda}\left(t^{1}, t^{2}, \ldots\right)=\left(f^{1}\left(t^{1}, \ldots, t^{m(1)} ; \lambda\right), f^{2}\left(t^{1}, \ldots, t^{m(2)} ; \lambda, \ldots\right)\right.
$$

with components $f^{i} \in \mathscr{F}$ defined for $\lambda \in \mathbb{R}$ close enough to zero, $|\lambda|<\varepsilon(i)$. Here $\varepsilon(i)>0$ but $\varepsilon(i) \rightarrow 0$ as $i \rightarrow \infty$ is admitted. So, rigorously speaking, $F_{\lambda}$ need not be a transformation if $\lambda \neq 0$; the above definition must be understood in the sense that an arbitrarily large number of coordinates of $F_{\lambda}(t)$ is well defined only for $\lambda$ sufficiently near to zero.

We shall suppose $F_{0}=$ identity in the sequel. Then, if the rule $F_{\lambda} \circ F_{\mu}=F_{\lambda+\mu}$ is satisfied, we shall speak of a (one-parameter, generalized, local) group. In more detail, we require the equalities

$$
\begin{aligned}
& f^{i}\left(f^{1}\left(t^{1}, \ldots, t^{m(1)} ; \mu\right), \ldots, f^{m(i)}\left(t^{1}, \ldots, t^{m(m(i))} ; \mu\right) ; \lambda\right) \equiv \\
& \equiv f^{i}\left(t^{1}, \ldots, t^{m(i)} ; \lambda+\mu\right)
\end{aligned}
$$

to hold whenever they make sense (for instance, if $t \in U^{\prime} \subset \boldsymbol{U} \subset \mathbb{R}^{\infty}$ and $|\lambda|,|\mu|<$ $\left.<\varepsilon\left(i, \boldsymbol{U}^{\prime}\right)\right)$. The vector field $Z=\sum^{\infty} z^{i}\left(\partial / \partial t^{i}\right)$ with $z^{i} \equiv \partial f^{i} /\left.\partial \lambda\right|_{\lambda=0}$ is called the infinitesimal transformation of the group $F_{\lambda}$ and we shall see that the relevant Lie system

$$
\partial f^{i}(\cdot ; \lambda) / \partial \lambda \equiv z^{i}\left(f^{1}(\cdot ; \lambda), \ldots, f^{m(i)}(\cdot ; \lambda)\right), \quad f^{i}(\cdot ; 0) \equiv t^{i}
$$

is of paramount importance since the Lie's first fundamental theorem can be carried over to our generalization and moreover, the family $\boldsymbol{G}$ of all infinitesimal transformations can be interestingly characterized.

6. Theorem. The group $F_{\lambda}$ satisfies $(*)$ and conversely, an arbitrary $F_{\lambda}$ satisfying (*) with certain functions $z^{i} \in \mathscr{F}$ is a group.

Proof. The direct assertion follows (as in the classical case) by applying $\partial / \partial \lambda$ to the composition rules at the value $\lambda=0$.

Conversely, let some general $F_{\lambda}$ satisfying $(*)$ be given. One can then verify that both $F_{\lambda+\mu}$ and $F_{\lambda} \circ F_{\mu}$ (with fixed $\mu$ ) are solutions of the differential equations of $(*)$ but with another (and in both cases the same) initial value $F_{\mu}$ at $\lambda=0$. In more detail, if we abbreviate

$$
\begin{aligned}
& F_{\lambda+\mu}\left(t^{1}, t^{2}, \ldots\right)=\left(F^{1}(\lambda), F^{2}(\lambda), \ldots\right), \\
& F_{\lambda \circ} F_{\mu}\left(t^{1}, t^{2}, \ldots\right)=\left(G^{1}(\lambda), G^{2}(\lambda), \ldots\right)
\end{aligned}
$$

with $t^{1}, t^{2}, \ldots$ mere parameters, then one can verify the identities

$$
\mathrm{d} y^{i} / \mathrm{d} \lambda \equiv z^{i}\left(y^{1}, \ldots, y^{m(i)}\right), \quad y^{i}(0) \equiv f^{i}\left(t^{1}, \ldots, t^{m(i)} ; \mu\right)
$$

for both families $y^{i} \equiv F^{i}$ and $y^{i} \equiv G^{i}$. So we need to prove the uniqueness property for the system $(* *)$ in order to be able to conclude $F^{i} \equiv G^{i}$ which expresses the desired composition rule. But $(* *)$ differs only inessentially from $(*)$ (namely in the choice of the initial value). So we need to prove uniqueness for the original system. 
Let us look at the first component $f^{1}$. By supposition, any change of the initial values $t^{k}(k>m(1))$ does not affect it, thus it does not affect the function $z^{1}=\partial f^{1} / \partial \lambda$. It follows that quite arbitrary functions $f^{k}(k>m(1))$ can be inserted into the system (*) with the result that the initial finite part of this system consisting of $m(1)$ equations for the remaining functions $f^{1}, \ldots, f^{m(1)}$ may have other solutions except the first component $f^{1}$ which is preserved (since $\partial f^{1} / \partial \lambda=z^{1}$ and $\left.f^{1}\right|_{\lambda=0}=t^{1}$ are). It follows that the first component $f^{1}$ of $F_{\lambda}$ can be calculated from a finite classical system of ordinary differential equations, so it is unique. (Other components $f^{i}$ are clearly unique, too.)

7. Theorem. A vector field $Z$ is an infinitesimal transformation (of some group $F_{\lambda}$ ) if and only if for every function $f \in \mathscr{F}$ the whole family of functions $f, Z f, Z^{2} f, \ldots$ can be expressed by a finite number of coordinates.

Proof. If $Z$ is an infinitesimal transformation of $F_{\lambda}$, then $Z^{j} f \circ F_{\lambda}=\mathrm{d}^{j}\left(f \circ F_{\lambda}\right) / \mathrm{d} \lambda^{j}$, hence $Z^{j} f=\mathrm{d}^{j}\left(f \circ F_{\lambda}\right) /\left.\mathrm{d} \lambda^{j}\right|_{\lambda=0}$ as follows from the composition rule (a classical argument). Consequently, for $f=f\left(t^{1}, \ldots, t^{n(f)}\right) \in \mathscr{F}$, all functions $Z^{j} f$ can be expressed by the coordinates $t^{1}, \ldots, t^{m(n(f))}$.

The inverse assertion is less trivial. Our first aim is to resolve $(*)$ without any additional assumption made on the vector field $Z$. Since the underlying space can be changed, the vector field $Z$ can be arbitrary modified outside a box containing the point under consideration. For instance, we may suppose each function $z^{j}=$ $=z^{j}\left(t^{1}, \ldots, t^{m(j)}\right)$ to be defined in all space $\mathbb{R}^{\infty}$ and to vanish if $\left|t^{1}\right|, \ldots,\left|t^{m(j)}\right|$ are large enough. After this adjustment, the functions $f_{k}^{i}(\lambda)(i, k=1,2, \ldots)$ determined by

$$
\begin{aligned}
& f_{k}^{i}(\lambda)=t^{i}(-1 / k \leqq \lambda \leqq 0), \\
& f_{k}^{i}(\lambda)=t^{i}+\int_{0}^{\lambda} z^{i}\left(f_{k}^{1}(\mu-1 / k), \ldots, f_{k}^{m(i)}(\mu-1 / k)\right) \mathrm{d} \mu \quad(\lambda \geqq 0)
\end{aligned}
$$

and depending on the parameters $t^{1}, t^{2}, \ldots$ are bounded and uniformly continuous. For a fixed $i$ they are even equicontinuous. So, owing to the Arzela-Ascoli theorem, there exist limits $f^{i}(\lambda)=\lim f_{k}^{i}(\lambda)$ for an appropriate sequence $k=k(1), k(2), \ldots \rightarrow$ $\rightarrow \infty$. The functions $f^{i}(\lambda)$ satisfy the original system $(*)$ only on a certain interval $0 \leqq \lambda<\varepsilon(i), \varepsilon(i)>0$, but it does not matter. Moreover, $f^{i}(\lambda)$ proves to be $C^{\infty}$ smooth in $\eta$ and as a function of the initial parameters $t^{1}, t^{2}, \ldots$ as follows by applying the common classical methods of the theory of ordinary differential equations. It is evident that the existence of solutions $f^{i}(\lambda)$ of $(*)$ on certain intervals $-\varepsilon(i)<\eta \leqq 0$ can be proved quite analogously and that the composed solution of $(*)$ on the interval $-\varepsilon(i)<\lambda<\varepsilon(i)$ is smooth. So the only fact to be verified is that every component $f^{i}$ depends only on a finite number of the parameters $t^{1}, t^{2}, \ldots$. At this place, the special assumption on the operator $Z$ is coming into play.

By successive differentiation of $\partial f^{i} / \partial \lambda=z^{i}$ and elimination of the derivatives $\partial f^{j} / \partial \lambda(j \neq i)$ with the use of $(*)$ one can obtain a sequence of identities

$$
\partial f^{i} / \partial \lambda=z_{1}\left(f^{1}, \ldots, f^{n(1)}\right), \partial^{2} f^{i} / \partial \lambda^{2}=z_{2}\left(f^{1}, \ldots, f^{n(2)}\right), \ldots
$$


where $z_{1}=z^{i}=Z t^{i}, z_{2}=Z z_{1}=Z^{2} t^{i}, \ldots$ By our assumption only a finite number of arguments $f^{1}, \ldots, f^{n}$ effectively appear in all these functions $z_{j}$. Let us now look at the finite system

$$
\mathrm{d} f^{i} / \mathrm{d} \lambda=z_{1}\left(f^{1}, \ldots, f^{n}\right), \ldots, \mathrm{d}^{n} f^{i} / \mathrm{d} \lambda^{n}=z_{n}\left(f^{1}, \ldots, f^{n}\right) .
$$

This is a finite system of ordinary differential equations with unknown functions $f^{1}, \ldots, f^{n}$, but the functions $f^{j}(j \neq i)$ are mere "parasite variables". If they are (locally) eliminated, at least one differential equation of order at most $n$ for the true unknown function $f^{i}$ appears. However, a solution of such an equation depends on at most $n$ initial values, the derivatives $\mathrm{d}^{k} f^{i} /\left.\mathrm{d} \lambda^{k}\right|_{\lambda=0}(k=0, \ldots, n-1)$. Returning to $(*)$ again, we can express every such derivative by a finite number of parameters $t^{1}, t^{2}, \ldots$ This concludes the proof.

8. A note on differential equations. A non-autonomous system $\mathrm{d} f^{i} / \mathrm{d} \lambda=$ $=g^{i}\left(\lambda, f^{1}, \ldots, f^{m(i)}\right)$ can be reduced to the form $(*)$ by introducing a new independent variable $\mu$ and denoting $\lambda=f^{0}$ with, say, $\mathrm{d} f^{0} / \mathrm{d} \mu=1$. Then all the above results can be applied. In particular, the linear system

$$
\mathrm{d} f^{i} / \mathrm{d} \lambda=a_{1}^{i}(\lambda) f^{1}+\ldots+a_{m(i)}^{i}(\lambda) f^{m(i)},\left.\quad f^{i}\right|_{\lambda=0}=c^{i}
$$

is of even greater interest since then the global existence and uniqueness can be proved. If, moreover, the coefficients $a_{j}^{i}(\lambda) \equiv a_{j}^{i}$ are constant, the criterion ensuring the inclusion $Z \in \boldsymbol{G}$ applied to the vector field $Z=\sum^{\infty} a_{i}^{j} t^{i}\left(\partial / \partial t^{j}\right)$ can be interestingly expressed in the following way: every fixed $j$-th row of all powers $A^{k}(k=1,2, \ldots)$ of the matrix $A=\left(a_{j}^{i}\right)$ is of limited length uniformly with respect to $k$. In geometric terms, if $A$ is identified with the transformation sending $t \in \mathbb{R}^{\infty}$ into $\bar{t} \in \mathbb{R}^{\infty}$ with $\bar{t}^{i}=\sum a_{j}^{i} t^{j}$, then all vectors $t, A t, A^{2} t, \ldots\left(t \in \mathbb{R}^{\infty}\right.$ is fixed here $)$ should be lying in a finite-dimensional linear subspace of $\mathbb{R}^{\infty}$. Such a transformation $A$ can be reduced to the Jordan normal form with the infinite number of diagonal blocks.

9. Regularity. Before continuing the main subject, we shall discuss some general concepts which will provide a comfortable link between algebra ( $\mathbb{R}$-linear spaces) and analysis $\left(\mathscr{F}\right.$-modules). Let $\mathscr{V}$ be an $\mathscr{F}$-module, $\mathscr{V}^{\wedge}$ the dual space consisting of all $\mathscr{F}$-linear $\mathscr{F}$-valued functions on $\mathscr{V}$. Then a basis of $\mathscr{V}$ (that is, $\mathscr{F}$-basis), weak $\left(\mathscr{F}\right.$-) basis of $\mathscr{V}^{\wedge}$, and the duality between them can be introduced quite analogously as in Section 3. (For instance, if $X_{1}, X_{2}, \ldots$ is a weak basis of $\mathscr{V}$, then every $X \in \mathscr{V}^{\wedge}$ can be expressed as $X=\sum^{\infty} x^{i} X_{i}$ with $x^{i}=\xi^{i}(X) \in \mathscr{F}$ where $\xi^{1}, \xi^{2}, \ldots$ is the relevant dual basis of $\mathscr{V}$. Conversely, every series $\sum^{\infty} x^{i} X_{i}\left(x^{i} \in \mathscr{F}\right)$ represents $X \in \mathscr{V}^{\wedge}$ determined by the values $\xi(X)=\sum x_{i} \xi^{i}(X) \in \mathscr{F}$ where the sum is finite.) The existence of bases of various $\mathscr{F}$-modules $\mathscr{V}$ will be tacitly assumed; then the weak bases of $\mathscr{V}^{\wedge}$ are determined by the duality.

Let $m_{t} \subset \mathscr{F}$ be the maximal ideal of all functions $f \in \mathscr{F}$ vanishing at a point $t \in U$. Let us denote $\mathscr{V}_{t}=\mathscr{V} / \Pi_{t} \mathscr{V}$ which is considered as an $\mathbb{R}$-linear space. We shall 
speak of a regular $\mathscr{F}$-module $\mathscr{V}$ if for every (equivalently: an appropriate) basis $\xi^{1}, \xi^{2}, \ldots$ of $\mathscr{V}$, the relevant classes $\xi_{t}^{1}, \xi_{t}^{2}, \ldots$ provide a basis of $\mathscr{V}_{t}$. If this is the case and $X_{1}, X_{2}, \ldots$ is a weak basis of $\mathscr{V}^{\wedge}$, then the relevant classes $\left(X_{1}\right)_{t},\left(X_{2}\right)_{t}, \ldots$ provide a weak basis of the $\mathbb{R}$-linear space $\mathscr{V}_{t}^{\wedge}=\mathscr{V}^{\wedge} / \mathrm{m}_{t} \mathscr{V}^{\wedge}$. We shall deal only with regular $\mathscr{F}$-modules. They have the nice property that many concepts can be "localized" at every point $t \in \boldsymbol{U}$. For instance, there is an isomorphism $\left(\mathscr{V}_{t}\right)^{\wedge}=$ $=\left(\mathscr{V}^{\wedge}\right)_{t}$ and every $\mathscr{F}$-homomorphism $L: \mathscr{V} \rightarrow \mathscr{W}$ between $\mathscr{F}$-modules induces $L_{t}: \mathscr{V}_{t} \rightarrow \mathscr{W}_{t}$ between the relevant $\mathbb{R}$-linear spaces, by factorization. Denoting by $\ell$ the cardinality of various bases (i.e., the dimension), the trivial equality $\ell(\mathscr{V})=\ell\left(\mathscr{V}_{t}\right)$ proves to be very useful.

The natural inclusion $\mathscr{U} \subset \mathscr{V}$ of a submodule $\mathscr{U}$ into a module $\mathscr{V}$ is called regular if every (equivalently: an appropriate) basis of $\mathscr{U}$ can be completed to a basis of $\mathscr{V}$. In this case, if $\mathscr{V}$ is regular then both $\mathscr{U}$ and $\mathscr{V} / \mathscr{U}$ are, too. Moreover, $\mathscr{U}^{\perp \perp}=\mathscr{U}$. (For a subset $\mathscr{A} \subset \mathscr{V}, \mathscr{A}^{\perp} \subset \mathscr{V}^{\wedge}$ consists of all $X \in \mathscr{V}^{\wedge}$ satisfying $\xi(X)=0$ for all $\xi \in \mathscr{A} ; \mathscr{U}^{\perp \perp}$ is defined analogously.) An $\mathscr{F}$-homomorphism $L: \mathscr{V} \rightarrow$ $\rightarrow \mathscr{W}$ between $\mathscr{F}$-modules will be called regular if the relevant inclusions Ker $L \subset \mathscr{V}$, Im $L \subset \mathscr{W}$ are regular in the above sense.

10. Differential forms and vector fields. The $\mathscr{F}$-module $\Phi=\Phi(\boldsymbol{U})$ of all differential forms $\xi=\sum x_{i} \mathrm{~d} t^{i}\left(x_{i} \in \mathscr{F}\right)$ is regular with the basis $\mathrm{d} t^{1}, \mathrm{~d} t^{2}, \ldots$ Then the dual $\mathscr{F}$-module $\Phi^{\wedge}$ has the dual weak basis $\partial / \partial t^{1}, \partial / \partial t^{2}, \ldots$ Let $\boldsymbol{G} \subset \Phi^{\wedge}$ be the subset of all infinitesimal transformations $Z$ of various groups $F_{\lambda}$. (Cf. Section 5 . At this place, we tacitly avoid some troubles concerning the domains of definition. In more exact terms, $\boldsymbol{G}$ should be defined as the set of all vector fields $Z$ which produce a group near every point of $\boldsymbol{U}$, that is, in an appropriate box.) Using the criterion of Theorem 7, one can easily see that $\boldsymbol{G}$ is a cone (i.e., $\mathscr{F} \boldsymbol{G} \subset \boldsymbol{G}$ ) but neither $\boldsymbol{G}+$ $+\boldsymbol{G} \subset \boldsymbol{G}$, nor $[\boldsymbol{G}, \boldsymbol{G}] \subset \boldsymbol{G}$ hold. (Hint: look at the vector fields $\sum^{\infty} t^{2 i+1} \partial / \partial t^{2 i}$, $\sum^{\infty} t^{2 i+2} \partial / \partial t^{2 i+1} \in \boldsymbol{G}$. Note that even $\left(\partial / \partial t^{1}\right)+\boldsymbol{G} \phi \boldsymbol{G}$ according to Section 11.) Let us state several properties (i) - (x) characterizing the vector fields $Z$ lying in $\boldsymbol{G}$ :

(i) For every $f \in \mathscr{F}$, the family $Z^{k} f(k=0,1, \ldots)$ is expressible by a finite number of coordinates (Theorem 7).

(ii) For every coordinate $t^{i}$, the family $Z^{k} t^{i}(k=0,1, \ldots)$ is expressible by a finite number of coordinates. ((i) $\Rightarrow$ (ii) is trivial, (ii) $\Rightarrow$ (i) follows from the formulae $\left.Z f=\sum\left(\partial f / \partial t^{i}\right) Z t^{i}, Z^{2} f=\sum\left(\partial^{2} f / \partial t^{i} \partial t^{j}\right) Z t^{i} Z t^{j}+\sum\left(\partial f / \partial t^{i}\right) Z^{2} t^{i}, \ldots\right)$

(iii) For every form $\xi \in \Phi$,' the family $\mathscr{L}_{\mathrm{Z}}^{k} \xi\left(k=0,1, \ldots ; \mathscr{L}_{\mathrm{Z}}=Z \neg \mathrm{d}+\mathrm{d}\right\urcorner Z$ is the Lie derivative) is expressible by a finite number of coordinates. ((iii) $\Rightarrow$ (i) follows by taking $\xi=\mathrm{d} f$, (i) $\Rightarrow$ (iii) follows by direct calculation with $\xi=\sum f^{i} \mathrm{~d} t^{i}$.)

(iv) For every form $\xi \in \Phi$, the family $\mathscr{L}_{Z}^{k} \xi(k=0,1, \ldots)$ is contained in a finitedimensional submodule of $\Phi$. ((iii) $\Rightarrow$ (iv) is trivial, (iv) $\Rightarrow$ (iii) follows by taking $\xi=\mathrm{d} f$ just as in point (iii).)

(v) For every term $\xi^{i}$ of a basis $\xi^{1}, \xi^{2}, \ldots$ of $\Phi$, the family $\mathscr{L}_{Z}^{k} \xi^{i}(k=0,1, \ldots)$ 
is contained in a finite-dimensional submodule of $\Phi$. ((iv) $\Rightarrow(\mathrm{v})$ is trivial, (v) $\Rightarrow$ (iv) follows by direct calculation of $\mathscr{L}_{\mathrm{Z}}^{k} \xi$ with $\xi=\sum x_{i} \xi^{i}$.)

(vi) For every term $\xi^{i}$ of a basis $\xi^{1}, \xi^{2}, \ldots$ of $\Phi$, the family $\mathscr{L}_{Z}^{k} \xi^{i}(k=0,1, \ldots)$ can be expressed by a finite number of coordinates. (Clearly (iv) $\Rightarrow$ (vi), (vi) $\Rightarrow(v)$.)

(vii) The same as (iii), (iv) but with the operator $\mathrm{dZ}\urcorner$ instead of $\mathscr{L}_{\mathrm{Z}}$. (We omit the proof and note that an analogous modification of (v), (vi) is not true.)

(viii) $-(\mathrm{x})$ : see Sections 11,12 and 16 .

11. Approximation of groups. Let us begin with a simplifying assumption, namely that $Z \in \boldsymbol{G}$ generates a uniform group, that is, the components $f^{i}$ of $F_{\lambda}(t)$ are defined for $|\lambda|<\varepsilon(\varepsilon \equiv \varepsilon(i)$ is independent of $i)$. Assume moreover that $Z$ is nonvanishing, for instance $z^{1}=\partial f^{1} / \partial \lambda \neq 0$. Then the function $g=g\left(t^{1}, \ldots, t^{m(1)}\right)$ satisfying $t^{1}\left(t^{1}, \ldots, t^{m(1)} ; g\right)=c$ with an appropriate and fixed once for all constant $c$ permits to introduce new coordinates

$$
\begin{aligned}
& \bar{t}^{1}=g\left(t^{1}, \ldots, t^{m(1)}\right), \\
& \bar{t}^{i}=f^{i}\left(t^{1}, \ldots, t^{m(i)} ; g\left(t^{1}, \ldots, t^{m(1)}\right)\right) \text { for } i>1 .
\end{aligned}
$$

Owing to $F_{-\lambda} \circ F_{\lambda}=$ identity, the inversions are $t^{i}=f^{i}\left(c, \bar{t}^{2}, \ldots, \bar{t}^{m(i)} ;-\bar{t}^{1}\right)$. Taking into account the rule $F_{\lambda} \circ F_{\mu}=F_{\lambda+\mu}$, one can verify that $F_{\lambda}^{*} \bar{t}^{1}=\bar{t}^{1}+\lambda, F_{\lambda}^{*} \bar{t}^{i}=\bar{t}^{i}$ $(i>1)$ so that $Z=\partial / \partial \bar{t}^{1}$ in the new coordinates.

Passing to a general $Z \in \boldsymbol{G}$ with, say, $z^{1} \neq 0$ as before, the above construction can be carried out only "approximatively" by taking

$$
\begin{aligned}
& \bar{t}^{1}=g\left(t^{1}, \ldots, t^{m(1)}\right), \\
& \bar{t}^{i}=f^{i}\left(t^{1}, \ldots, t^{m(i)} ; g\right) \text { for } 1<i \leqq M, \quad \bar{t}^{i}=t^{i} \text { for } i>M,
\end{aligned}
$$

where $M$ is fixed and and very big (to ensure some properties needed later on). The existence of a certain inversion $t^{i}=g^{i}\left(\bar{t}^{1}, \ldots, \bar{t}^{n(i)}\right)$ follows by the classical implicit function theorem (since the system $\bar{t}^{i}=f^{i}(1<i \leqq M)$ reduces to the identity $\bar{t}^{i} \equiv t^{i}$ if $\left.t^{1}=c\right)$. It is clear that even $g^{i}\left(t^{1}, \ldots, t^{n(i)}\right)=f^{i}\left(c, t^{2}, \ldots, t^{m(i)} ;-t^{1}\right)$ for as many indices $i$ as desired, by an appropriate choice of a very big $M$. Moreover, one can ensure that $F_{\lambda}^{*} \bar{t}^{1}=\bar{t}^{1}+\lambda$ and $F_{\lambda}^{*} \bar{t}^{i}=\bar{t}^{i}$ for arbitrarily many indices $i$, e.g., for $i \leqq N$. It follows that $Z=\partial / \partial \bar{t}^{1}+R_{N}$ where $R_{N}$ is a series involving only the summands $\partial / \partial \bar{t}^{k}$ with $k>N$. Every finite family $g^{1}, \ldots, g^{c} \in \mathscr{F}$ can be expressed by the variables $\bar{t}^{1}, \ldots, \bar{t}^{N}$ if $N$ is chosen big enough. So it follows that $Z^{k} g^{i}=$ $=\partial^{k} g^{i} /\left(\partial \bar{t}^{1}\right)^{k}$ for $k=0,1, \ldots$ in an appropriate coordinate system. (Conversely, the latter property noted (viii) of a vector field $Z \in \Phi^{\wedge}$ clearly ensures $Z \in G$, even in the weakened form $c=1$.) Still in other terms: the above results mean that the uniform group $F_{\lambda}^{\prime}$ defined by $F_{\lambda}^{\prime}\left(\bar{t}^{1}, \bar{t}^{2}, \bar{t}^{3}, \ldots\right)=\left(\bar{t}^{1}+\lambda, \bar{t}^{2}, \bar{t}^{3}, \ldots\right)$ approximates the original group $F_{\lambda}$ in the sense that both $F_{\lambda}, F_{\lambda}^{\prime}$ operate in the same manner on as many coordinates as one needs, $f^{i}\left(t^{1}, \ldots, t^{m(i)} ; \lambda\right)=f^{\prime i}\left(t^{1}, \ldots, t^{m(i)} ; \lambda\right)$ for a large number of indices $i$. So if we deal with a finite number of functions, the action of 
a group $F_{\lambda}$ can be replaced by the action of an appropriate uniform group $F_{\lambda}^{\prime}$ without any change of the result.

12. Towards the Frobenius theorem. Let $Z \in \Phi^{\wedge}$ be a nonvanishing vector field. Denote by $Z^{\perp}$ the submodule of $\Phi$ consisting of all forms $\xi \in \Phi$ satisfying $\xi(Z)=0$. Such a $Z$ is an infinitesimal transformation of a uniform group $F_{\lambda}$ if and only if there is a basis of $Z^{\perp}$ consisting of total differentials.

Proof. If $Z$ generates a uniform group $F_{\lambda}$, then $Z=\partial / \partial \bar{t}^{1}$ in appropriate coordinates $\bar{t}^{1}, \bar{t}^{2}, \ldots$ and $\mathrm{d} \bar{t}^{2}, \mathrm{~d} \bar{t}^{3}, \ldots$ is a basis of $Z^{\perp}$. Conversely, if $\mathrm{d} h^{1}, \mathrm{~d} h^{2}, \ldots$ is a basis of $Z^{\perp}$ and $h=h^{0} \in \mathscr{F}$ is a function with $Z h \neq 0$, then $h^{0}, h^{1}, \ldots$ may be used for coordinates so that $Z=Z h \partial / \partial h$ clearly generates a uniform group.

More generally, $Z \in \boldsymbol{G}$ if and only if every $\xi \in Z^{\perp}$ can be expressed as a linear combination of some linearly independent total differentials lying in $Z^{\perp}$.

Proof. Let $Z \in \boldsymbol{G}$ and $\xi=z_{1} \mathrm{~d} t^{1}+\ldots+z_{n} \mathrm{~d} t^{n} \in \Phi$. We may ensure $Z=$ $=\partial / \partial \bar{t}^{1}+R_{N}$ in some new coodinates, and suppose that $t^{1}, \ldots, t^{n}$ can be expressed. by $\bar{t}^{1}, \ldots, \bar{t}^{N}$ and $R_{N}$ is a series involving only $\partial / \partial \bar{t}^{i}(i>N)$. Then the inclusion $\xi \in Z^{\perp}$ implies $\xi \in\left\{\mathrm{d} \bar{t}^{1}, \ldots, \mathrm{d} \bar{t}^{N}\right\} \subset Z^{\perp}$. Conversely, let the above property (briefly: property (ix)) be satisfied for every form $\xi \in Z^{\perp}$. Let $\xi^{2}, \xi^{3}, \ldots$ be a basis of the $\mathscr{F}$-module $Z^{\perp}$. Then we may write $\xi^{i}=\sum g_{j} \mathrm{~d} h^{j}$ with some $\mathrm{d} h^{j} \in Z^{\perp}$ (property (ix)). One can easily verify $\mathscr{L}_{Z}^{k} \xi^{i}=(Z \neg \mathrm{d})^{k} \xi^{i}=\sum Z^{k} g_{j} \mathrm{~d} h^{j}$ so that the family $\mathscr{L}_{Z}^{k} \xi^{i}$ ( $i$ is fixed but $k=0,1, \ldots$ ) is lying in a finite-dimensional submodule of $\Phi$. We wish to apply the criterion (v) of section 10 to conclude $Z \in G$; however, we still have no basis of $\Phi$. Let $f \in \mathscr{F}$ satisfy $Z f \neq 0$. Replacing $Z$ by $Z / Z f$, we may suppose $Z f=1$. Denoting $\xi^{1}=\mathrm{d} f$, we have $\mathscr{L}_{Z} \xi^{1}=0$ so that the above mentioned criterion can be applied to the basis $\xi^{1}, \xi^{2}, \ldots$ of $\Phi$.

One can discern the above two results as a simple variant of the famous Frobenius theorem for the space $\mathbb{R}^{\infty}$ in the particular case of one-dimensional submodules of $\Phi^{\wedge}$ (i.e., of nonvanishing vector fields). For the general case, it is desirable to employ the tools of differential forms to greater extent.

Let $\Omega \subset \Phi$ be a submodule with the regular inclusion. We shall denote $\mathscr{H}=$ $=\Omega^{\perp} \subset \Phi^{\wedge}$, hence $\mathscr{H}^{\perp}=\Omega$. The module $\Omega$ is called formally integrable (briefly: flat) if the equivalent conditions

$$
[\mathscr{H}, \mathscr{H}] \subset \mathscr{H}, \quad \mathscr{H} \neg \mathrm{d} \Omega \subset \Omega, \quad \mathscr{L}_{\mathscr{H}} \Omega \subset \Omega, \mathrm{d} \Omega=0 \text { modulo } \Omega
$$

are satisfied. If, moreover, $\ell(\Omega)<\infty$, then the common Frobenius theorem applies and we conclude that $\Omega$ has a basis consisting of total differentials, and consequently $\mathscr{H}$ admits a weak basis with terms in $\boldsymbol{G}$. Passing to the less trivial case $\ell(\Omega)=\infty$, we may state the following generalizations of the above results (in which we had $\Omega=Z^{\perp}$ and $\left.\ell(\mathscr{H})=1\right)$.

13. Theorem. Let $\Omega$ be flat and $\ell(\mathscr{H})<\infty$. Then every $Z \in \mathscr{H}$ is an infinitesimal transformation of a uniform group if and only if there exists a basis of $\Omega$ consisting of total differentials. 
We omit the proof prefering the more interesting

14. Theorem. Let $\Omega$ be flat and $\ell(\mathscr{H})<\infty$. Then $\mathscr{H} \subset \boldsymbol{G}$ if and only if every form $\omega \in \Omega$ can be expressed as a linear combination of some linearly independent total differentials lying in $\Omega$.

Proof. Using a slightly different notation than usually, we shall assume that $\mathrm{d} x^{1}, \ldots, \mathrm{d} x^{n}, \mathrm{~d} y^{1}, \mathrm{~d} y^{2}, \ldots$ is a basis of $\Phi$ where $\mathrm{d} x^{1}, \ldots, \mathrm{d} x^{n}(n=\ell(\mathscr{H}))$ provide a basis of $\Phi / \Omega$. Then certain forms $\omega^{j}=\mathrm{d} y^{j}-\sum y_{i}^{j} \mathrm{~d} x^{i}(j=1,2, \ldots)$ provide a basis of $\Omega$, and then the vector fields $Z_{i}=\partial / \partial x^{i}+\sum y_{i}^{j} \partial / \partial y^{j}(i=1, \ldots, n)$ may serve as a basis of $\mathscr{H}$.

First, assume $\mathscr{H} \subset \boldsymbol{G}$. Since the case $\ell(\mathscr{H})=1$ is already clear, we shall proceed by induction. It is sufficient to consider only the form $\omega=\omega^{j}$ with a fixed $j$. Let $Z \in \mathscr{H}$. We may assume $Z=Z_{1}$, hence $Z=\partial / \partial x^{1}$. (Use the approximation of section 11 with coordinates $t^{i}=-x^{i}(i=1, \ldots, n)$ and $t^{n+j}=y^{j}(j=0,1, \ldots)$, and realize that $Z$ will be applied on a finite family of functions in the rest of the proof.) Then $\omega^{j}(Z)=0 \quad$ implies $\quad y_{1}^{j}=0, \quad \mathscr{L}_{Z} \omega^{j}=Z \neg \mathrm{d} \omega^{j}=\sum\left(\partial y_{i}^{j} / \partial x^{1}\right) \mathrm{d} x^{i} \in \Omega$ implies $\partial y_{i}^{j} / \partial x^{1}=0$, thus the form $\omega^{j}$ is independent of the variable $x^{1}$. We pass to the induction. The form $\omega^{j}$ restricted to $x^{1}=$ const. can be expressed as $\omega^{j}=\sum g_{k} \mathrm{~d} h^{k}$ where $\mathrm{d} h^{k}$ lie in the restriction of $\Omega$ on $x^{1}=$ const. When $g_{k}, h^{k}$ are regarded as functions on the whole space but independent of the coordinate $x^{1}$, then the above expression of $\omega^{j}$ is still valid and, moreover, $\mathrm{d} h^{k} \in \Omega$. (The last inclusion follows from $Z_{1} h^{k}=Z h^{k}=\partial h^{k} / \partial x^{1}=0$ and for $i=2, \ldots, n$ we have $\partial\left(Z_{i} h^{k}\right) / \partial x^{1}=Z Z_{i} h^{k}=$ $=Z_{i} Z h^{k}+\left[Z, Z_{i}\right] h^{k}=0$ (since $\left.\left[Z, Z_{i}\right]=\left[Z_{1}, Z_{i}\right]=0\right)$ and $Z_{i} h^{k}=0$ on $x^{1}=$ = const. Hence $Z_{i} h^{k} \equiv 0$.)

Conversely, let $f \in \mathscr{F}, Z=\sum f^{i}\left(\partial / \partial x^{i}\right)+\sum^{\infty}\left(g^{j} \partial / \partial y^{j}\right) \in \mathscr{H}$, where $f, f^{1}, \ldots, f^{n}$ are functions expressible by the variables $x^{1}, \ldots, x^{n}, y^{1}, \ldots, y^{M}$. According to the assumption of the theorem we suppose $\omega^{1}, \ldots, \omega^{M} \in\left\{\mathrm{d} h^{1}, \ldots, \mathrm{d} h^{c}\right\} \subset \Omega$. Let us include the functions $h^{1}, \ldots, h^{c}$ into the family of coordinates instead of the appropriate original coordinates $y^{j}$. We obtain, for instance, the new coordinates $x^{1}, \ldots, x^{n}$, $h^{1}, \ldots, h^{c}, y^{c+1}, y^{c+2}, \ldots$. In terms of these new coordinates we have $Z=$ $=\sum f^{i} \partial / \partial x^{i}+\sum^{\infty} g^{j} \partial / \partial y^{j} \quad$ (the second sum with $\left.j=c+1, c+2, \ldots\right)$ and all functions $Z^{k} f=\left(\sum f^{i} \partial / \partial x^{i}\right)^{k} f$ are clearly expressible by the coordinates $x^{1}, \ldots, x^{n}$, $x^{1}, \ldots, h^{c}$.

15. A note on generalization. Assume $\Omega$ is flat and the dimension $\ell(\mathscr{H})$ arbitrary. Let $x^{1}, x^{2}, \ldots, y^{1}, y^{2}, \ldots \in \mathscr{F}$ be functions such that their differentials $\mathrm{d} x^{1}, \mathrm{~d} x^{2}, \ldots$ $\ldots, \mathrm{d} y^{1}, \mathrm{~d} y^{2}, \ldots$ provide a basis of $\Phi$ and at the same time $\mathrm{d} x^{1}, \mathrm{~d} x^{2}, \ldots$ provide a basis of $\Phi / \Omega$. Every $Z \in \Phi^{\wedge}$ can be represented as $Z=\sum^{(\infty)} f^{i} \partial / \partial x^{i}+\sum^{(\infty)} g^{j} \partial / \partial y^{j}=$ $=X+Y$. Then the following generalization of Theorem 14 can be established: every form $\omega \in \Omega$ can be expressed as a linear combination of certain differentials from $\Omega$ if and only if, for every $Z \in \mathscr{H}$, the inclusion $X \in \boldsymbol{G}$ implies $Z \in \boldsymbol{G}$. Since this result looks rather ugly, we omit the proof (which does not bring much new 
ideas). For the case $\ell(\mathscr{H})<\infty$, the inclusion $X \in \boldsymbol{G}$ is always valid and the assertion reduces to Theorem 14 .

16. The Monge systems of equations. Let us introduce the following property (x) of a vector field $Z \in \Phi^{\wedge}$ : for every $\zeta \in Z^{\perp}$, the family $\mathscr{L}_{Z}^{k} \zeta(k=0,1, \ldots)$ generates a finite-dimensional submodule of $\Phi$ (in reality a submodule of $Z^{\perp}$ since $\xi \in Z^{\perp}$ always implies $\mathscr{L}_{\mathbf{Z}} \xi=Z \neg \mathrm{d} \xi \in Z^{\perp}$ ). One can easily see that (x) is equivalent to the inclusion $Z \in \boldsymbol{G}$ (cf. Theorem 14 or, directly, look at the end of the proof in Section 12). On the other hand, let $\boldsymbol{F} \subset \Phi^{\wedge}$ be the subset of all $Z \in \Phi^{\wedge}$ for which there exists a finite set of forms $\zeta^{1}, \ldots, \zeta^{c} \in Z^{\perp}$ such that the family of forms $\mathscr{L}_{Z}^{k} \zeta^{j}(k=0,1, \ldots$; $j=1,2, \ldots)$ may be used for generators of the $\mathscr{F}$-module $Z^{\perp}$. (According to (c), $\boldsymbol{F}$ may be considered as an "opposite object" to $\boldsymbol{G}$.) One can easily see that $f \boldsymbol{F} \subset \boldsymbol{F}$ for every nonvanishing $f \in \mathscr{F}$.

We pass to an interpretation of vector fields from $\boldsymbol{F}$. Let $Z \in \boldsymbol{F}$ and $x \in \mathscr{F}$ with $Z x \neq 0$. Replacing $Z$ by $Z / Z f \in \boldsymbol{F}$ we may suppose $Z x=1$. Let the forms $\zeta^{1}, \ldots, \zeta^{c}$ from the above definition be all expressible by the variable $x$ and appropriate functions $y^{1}, \ldots, y^{m}$. Denoting $y_{0}^{j} \equiv y^{j}$ and taking recurrently $y_{k+1}^{j}=Z y_{k}^{j}$, one can verify that $\omega_{k}^{j}=\mathrm{d} y_{k}^{j}-y_{k+1}^{j} \mathrm{~d} x \in Z^{\perp}$ and $\mathscr{L}_{Z} \omega_{k}^{j}=\omega_{k+1}^{j}$. The forms $\omega_{k}^{j}$ generate the module $Z^{\perp}$.

It may happen that these forms $\omega_{k}^{j}$ are linearly independent. Then they provide a basis of $Z^{\perp}$, the family of all differentials $\mathrm{d} x, \mathrm{~d} y_{k}^{j}$ may serve for a basis of $\Phi$, and the functions $x, y_{k}^{j}$ may be taken for coordinates. We enter the area of the jet theor $y$ of curves (all curves $y^{j}=y^{j}(x) ; j=1, \ldots, m$; in the space $\mathbb{R}^{m+1}$ ): the coordinates $y_{k}^{j}$ stand for the derivatives $\mathrm{d}^{k} y^{j} / \mathrm{d} x^{k}, \omega_{k}^{j}$ are identical with the familiar contact forms and, in terms of new coordinates, $Z=\partial / \partial x+\sum^{\infty} y_{k+1}^{j} \partial / \partial y_{k}^{j}$ is the total derivative in the direction of the curves. However, such an identification is possible only as a result of a very successful choice of the initial variables $x, y^{1}, \ldots, y^{m}$. It is a very nontrivial problem how to do it.

In general, the forms $\mathrm{d} x, \mathrm{~d} y^{1}, \ldots, \mathrm{d} y^{m}$ may be assumed linearly independent, and then the family $\omega^{1}, \ldots, \omega^{m}$ is independent, too. But there may exist some relations $\left(R_{1}\right)$

$$
y_{1}^{l}=g_{1}^{l}\left(x, y^{1}, \ldots, y^{m}, y_{1}^{1}, \ldots, y_{1}^{\mu_{1}}\right), \quad l=\mu_{1}+1, \ldots, m,
$$

between the variables $x, y^{1}, \ldots, y^{m}, y_{1}^{1}, \ldots, y_{1}^{m}$ and consequently, between the forms $\omega_{0}^{1}, \ldots, \omega_{0}^{m}, \omega_{1}^{1}, \ldots, \omega_{1}^{m}$. (We have

$$
\left.\omega_{1}^{l}=\sum\left(\partial g_{1}^{l} / \partial y^{j}\right) \omega_{0}^{j}+\sum\left(\partial g_{1}^{l} / \partial y_{1}^{k}\right) \omega_{1}^{k} .\right)
$$

Moreover, besides the derived relations $\omega_{2}^{l}=Z y_{1}^{l}=Z g_{1}^{l}\left(l=\mu_{1}+1, \ldots, m\right)$, there may exist others, for instance

$\left(R_{2}\right) \quad y_{2}^{l}=g_{2}^{l}\left(x, y^{1}, \ldots, y^{m}, y_{1}^{1}, \ldots, y_{1}^{\mu_{1}}, y_{2}^{1}, \ldots, y_{2}^{\mu_{2}}\right), \quad l=\mu_{2}+1, \ldots, \mu_{1}$,

and so on. So we obtain the basis 


$$
\mathrm{d} x, \mathrm{~d} y_{0}^{1}, \ldots, \mathrm{d} y_{0}^{\mu_{0}}, \mathrm{~d} y_{1}^{1}, \ldots, \mathrm{d} y_{1}^{\mu_{1}}, \ldots \quad\left(y_{0}^{j} \equiv y^{j}, \mu_{0}=m\right)
$$

of the module $\Phi$, and the basis $\omega_{0}^{1}, \ldots, \omega_{0}^{\mu_{0}}, \omega_{1}^{1}, \ldots, \omega_{1}^{\mu_{1}}, \ldots$ of $Z^{\perp}$. Clearly $Z=$ $=\partial / \partial x+\sum^{\infty} y_{k+1}^{l} \partial / \partial y_{k}^{l}$ as before but the sum is taken only through $j=1, \ldots, \mu_{k}$ (and $k=0,1, \ldots$ ). Since $\mu_{0}=m \geqq \mu_{1} \geqq \mu_{2} \geqq \ldots$, necessarily $\mu_{k} \equiv \mu$ is constant for all $k$ large enough (so that no new relations $R_{k}$ appear if $k$ is big enough). In classical terms, where $y_{k}^{j}$ are interpreted as derivatives, we have the (finite) Monge system of ordinary differential equations $y_{k}^{l}=g_{k}^{l}\left(k=1,2, \ldots ; l=\mu_{k}+1, \ldots, \mu_{k-1}\right)$ or better, its infinite prolongation since all derived relations $y_{k+j}^{l}=Z^{j} g_{k}^{l}$ are also taken into account. It is to be pointed out that such an interpretation appears only after the choice of the initial variables so that the vector field $Z \in \boldsymbol{F}$ represents the Monge system "abstractly" without the presence of any additional and accidental structure.

17. A note on the number of generators. It may be proved that the above constant $\mu=\mu(\Omega)$ depends only on the vector field $Z$ under consideration, or better, on the module $Z^{\perp}=\Omega$. On the contrary, the number $c$ of the generating forms $\zeta^{1}, \ldots, \zeta^{c}$ is not of this kind. Nonetheless, we may introduce an intrinsical constant $\varrho=\varrho(\Omega)$ as the minimum of all possible numbers $c$ of generators.

If we deal with all curves in the space $\mathbb{R}^{m+1}$, that is, with the module $\Omega$ of all contact forms $\omega_{k}^{j}(k=0,1, \ldots ; j=1, \ldots, m)$, then clearly $\varrho(\Omega)=m$ and $c=m$ (choose $\zeta^{j}=\omega_{0}^{j}, j=1, \ldots, m$, for the generators). Since always $\varrho \geqq \mu$, we conclude $\varrho=m=\mu$ for the module of contact forms.

This example seems to be very suggestive and the conjecture may arise that the equality $\varrho(\Omega)=\mu(\Omega)$ is a typical property of the module of contact forms. However, this is not the case even if $\varrho(\Omega)=\mu(\Omega)=1$. The point lies in the fact that the generating form may be rather complicated. (Example. Let the coordinates be denoted by $x, u, v, w_{0}, w_{1}, \ldots$ and let the module $\Omega$ be generated by the forms $\xi=\mathrm{d} u-f \mathrm{~d} x$, $\eta=\mathrm{d} y-g \mathrm{~d} x, \quad \vartheta_{i}=\mathrm{d} w_{i}-w_{i+1} \mathrm{~d} x \quad(i=0,1, \ldots)$, where $f=f\left(x, w_{1}\right), g=$ $=g\left(x, w_{1}\right)$ are given functions. Clearly $\Omega=Z^{\perp}$ where $Z=\partial / \partial x+f \partial / \partial u+$ $+g(\partial / \partial v)+\sum^{\infty} w_{k+1}\left(\partial / \partial w_{k}\right) \in \boldsymbol{F}$. If we take $\zeta=a \vartheta_{0}+b \xi+c \eta$ with coefficients satisfying

$$
a+b \frac{\partial f}{\partial w_{1}}+c \frac{\partial g}{\partial w_{1}}=a+b Z\left(\frac{\partial f}{\partial w_{1}}\right)+c Z\left(\frac{\partial g}{\partial w_{1}}\right)=0,
$$

then

$$
\mathscr{L}_{Z} \zeta=Z a \vartheta_{0}+Z b \xi+Z c \eta, \quad \mathscr{L}_{Z}^{2 \zeta}=Z^{2} a \vartheta_{0}+Z^{2} b \xi+Z^{2} c \eta
$$

which permits to express the forms $\vartheta_{0}, \xi, \eta$ in terms of $\zeta, \mathscr{L}_{Z} \zeta, \mathscr{L}_{Z}^{2} \zeta$. Then, using the operator $\mathscr{L}_{\mathrm{Z}}$, all forms $\vartheta_{k}$ can be expressed in terms of $\zeta, \mathscr{L}_{\mathrm{Z}} \zeta, \ldots$ so that $\zeta^{1}=\zeta$ can be taken for the generating form and $\varrho(\Omega)=1$. On the other hand, it can be proved that the module $\Omega$ is quite different from the module of all contact forms 
(to the curves in $\mathbb{R}^{2}$ ) provided the functions $f, g$ are general enough. Indeed, if

$$
\operatorname{det}\left(\begin{array}{lll}
\partial^{2} f / \partial w_{1}^{2} & \partial^{2} f / \partial x & \partial w_{1} \\
\partial^{2} g / \partial w_{1}^{2} & \partial^{2} g / \partial x & \partial w_{1}
\end{array}\right) \neq 0,
$$

then $\Omega$ is not explicitly solvable.)

18. Diffietics. A flat submodule $\Omega \subset \Phi$ is called a diffiety if there exist forms $\zeta^{1}, \ldots, \zeta^{c}$ such that the family $\mathscr{L}_{Z}^{k} \zeta^{j}(Z \in \mathscr{H} ; k=0,1, \ldots ; j=1, \ldots, c)$ generates the $\mathscr{F}$-module $\Omega$; here $\mathscr{H}=\Omega^{\perp}$ and we suppose $\ell(\mathscr{H})=n<\infty$. For the particular case $\ell(\mathscr{H})=1$, we may use only the family of generators $\mathscr{L}_{Z}^{k} \zeta^{j}$ where $Z \in \mathscr{H}$ is a fixed nonvanishing vector field and the above definition means exactly that $Z \in F$. So, according to Section 16 , a diffiety with $\ell(\mathscr{H})=1$ may be identified with the infinite prolongation of a system of ordinary differential equations. Quite analogously, a general diffiety $\Omega$ may be identified with the infinite prolongation of a system of partial differential equations with $n=\ell(\mathscr{H})$ independent variables. In general $\mathscr{H} \notin \boldsymbol{F}$. Even the intersection $\mathscr{H} \cap \boldsymbol{G}$ may be nonempty and then it determines the Cauchy characteristics. The true meaning of $\mathscr{H} \cap \boldsymbol{F}$ is not yet known.

19. Infinitesimal symmetries. A vector field $S \in \Phi^{\wedge}$ is called a generalized (or: Lie-Bäcklund) infinitesimal symmetry of a diffiety $\Omega$ if $\mathscr{L}_{S} \Omega \subset \Omega$ or, equivalently, $[S, \mathscr{H}] \subset \mathscr{H}$. (The equivalence follows from the identity $0=\mathscr{L}_{S}(Z \neg \omega)=$ $=[S, Z] \neg \omega+Z \neg \mathscr{L}_{S} \omega$ with $Z \in \mathscr{H}$ and $\omega \in \Omega$.) If, moreover, $Z \in \boldsymbol{G}$, then the relevant one-parameter group $F_{i}$ preserves $\Omega$ and $\mathscr{H}$ in the obvious sense. In general, a symmetry $S \notin \boldsymbol{G}$ cannot be related with any reasonable automorphism of the underlying space. However, it can be identified with use a certain special kind of diffiety $\bar{\Omega}$ in an extended space (endowed by certain additional structures), the so called evolution diffiety (to $\Omega$ ).

This is done as follows. Besides the original coordinates $t^{1}, t^{2}, \ldots$ we introduce a new coordinate $t^{0}$ to obtain the points $\bar{t}=\left(t^{0}, t^{1}, \ldots\right)$ of the extended space $\bar{U}=$ $=\mathbb{P} \times \boldsymbol{U}$ instead of the original points $t=\left(t^{1}, t^{2}, \ldots\right) \in \boldsymbol{U} \subset \mathbb{R}^{\infty}$. Using the common convention, we identify $\mathscr{F}=\mathscr{F}(U) \subset \overline{\mathscr{F}}=\mathscr{F}(\bar{U})$ with the submodules, and analogously $\Phi \subset \bar{\Phi}=\Phi(\bar{U})$ and even $\Phi^{\wedge} \subset \bar{\Phi}^{\wedge}$ (the horizontal and projectable vector fields). Then, if a diffiety $\Omega$ and a vector field $S \in \Phi^{\wedge}$ are given, we introduce the $\overline{\mathscr{F}}$-module $\bar{\Omega} \subset \bar{\Phi}$ generated by all forms $\omega-\omega(S) \mathrm{d} t^{0}(\omega \in \Omega)$ so that $\overline{\mathscr{H}}=$ $=\bar{\Omega}^{\perp} \subset \bar{\Phi}^{\perp}$ is generated by the vector field $\bar{Z}=\partial / \partial t^{0}+S \in \bar{\Phi}^{\wedge}$ and all vector fields $Z \in \mathscr{H}\left(\subset \Phi^{\wedge} \subset \bar{\Phi}^{\wedge}\right)$. One can then verify that such $\bar{\Omega}$ is flat (and hence a diffiety, see the next section) if and only if $S$ is a generalized symmetry of $\Omega$.

So the generalized symmetries may be identified with the evolutional diffieties A huge number of articles deal with generalized symmetries of evolutional diffieties (that is, with the simultaneous theory of two symmetries), especially if the original diffiety $\Omega$ is the module of all contact forms to the curves in $\mathbb{R}^{2}$. But even in the simplest cases, the search for all such symmetries proves to be a toilsome task as yet resolved only case by case. 
20. Continuation. Every field $S \in \mathscr{H}$ is a generalized symmetry of $\Omega=\mathscr{H}^{\perp}$. However, the existence of nontrivial symmetries $S \notin \mathscr{H}$ proves to be a very restrictive requirement. For this reason the following weakened concept may be of certain interest: a flat submodule $\Omega^{\prime} \subset \Phi$ is called an overdiffiety of a diffiety $\Omega$ if $\Omega^{\prime} \subset \Omega$ (that is, if $\mathscr{H}^{\prime}=\Omega^{\perp \perp} \supset \mathscr{H}=\Omega^{\perp}$ which means that "solutions of $\Omega$ lie in solutions of $\left.\Omega^{\prime} "\right)$. Note aside that the higher is $\ell\left(\Omega / \Omega^{\prime}\right)$, the easier is the calculation of overdiffieties. The case $\ell\left(\Omega / \Omega^{\prime}\right)=1$ is closely related to generalized symmetries.

For brevity we shall deal only with the latter case. Then $\mathscr{H}^{\prime}$ is generated by $\mathscr{H}$ and one additional vector field $Y \notin \mathscr{H}$, hence $\Omega^{\prime}=Y^{\perp} \cap \Omega$. The flatness of $\Omega^{\prime}$ may be expressed by $[Y, \mathscr{H}] \subset \mathscr{H}^{\prime}$ or, in dual terms, by

$$
\mathscr{L}_{\mathbf{Y}}\left(Y^{\perp} \cap \Omega\right) \subset Y^{\perp} \cap \Omega \quad(Y \notin \mathscr{H}) .
$$

It is interesting to note that such a flat $\Omega^{\prime}$ is in reality a diffiety. (Proof. Let $\zeta^{1}, \ldots, \zeta^{c} \in \Omega$ be forms giving the generators of the $\mathscr{F}$-module $\Omega$ after the repeating application of the operators $\mathscr{L}_{Z}(Z \in \mathscr{H})$. We may assume $\zeta^{1}(Y)=1, \zeta^{2}(Y)=\ldots$ $\ldots=\zeta^{c}(Y)=0$, hence $\zeta^{2}, \ldots, \zeta^{c} \in \Omega^{\prime}$ and so $\mathscr{L}_{\mathscr{H}^{\prime}}^{k} \zeta^{j}(j=2, \ldots, c)$. As the form $\zeta^{1}$ is concerned, we have

$$
\left.\zeta_{j}=\mathscr{L}_{Z_{j}} \zeta^{1}-f_{j} \zeta^{1} \in Y^{\perp} \cap \Omega=\Omega^{\prime} \quad\left(f_{j}=Y\right\urcorner \mathscr{F}_{Z} \zeta^{1}=\mathrm{d} \zeta^{1}\left(Y, Z_{j}\right)\right)
$$

for every $j=1, \ldots, n$, where $Z_{1}, \ldots, Z_{n}$ is a basis of $\mathscr{H}$. It follows that even the repeating use of the operators $\mathscr{L}_{Z}(Z \in \mathscr{H})$ on the forms $\zeta_{1}, \ldots, \zeta_{n}, \zeta^{2}, \ldots, \zeta^{c}$ produces the family of generators of the $\mathscr{F}$-module $\Omega^{\prime}$.) Now the point of the construction lies in the obvious fact that for every nontrivial generalized symmetry $S \notin \mathscr{H}$ of $\Omega$, the module $\Omega^{\prime}=S^{\perp} \cap \Omega$ is an overdiffiety of $\Omega$.

In explicit terms, let $\Omega^{\prime}=Y^{\perp} \cap \Omega$ be an overdiffiety of $\Omega$. We ask whether there exists $S \in \mathscr{H}^{\prime}$ with $\Omega^{\prime}=S^{\perp} \cap \Omega$ and $[S, \mathscr{H}] \subset \mathscr{H}$. Since such a vector field $S$ may be replaced by every $S+Z(Z \in \mathscr{H})$, we may find $S$ in the form $S=f Y$ for an appropriate $f \in \mathscr{F}$. The resulting equation $[f Y, \mathscr{H}] \subset \mathscr{H}$ can be made more transparent if we use a basis $Z_{1}, \ldots, Z_{n}$ of $\mathscr{H}$ and introduce $h_{1}, \ldots, h_{n}$ satisfying $\left[Y, Z_{i}\right]=$ $=h_{i} Y$ modulo $\mathscr{H}$. Then we obtain the conditions $\left[f Y, Z_{i}\right] \equiv\left(f h_{i}-Z_{i} f\right) Y \in \mathscr{H}$, that is

$$
Z_{i} f=f h_{i} \quad(i=1, \ldots, n) .
$$

The same conditions in dual terms (advantageous for calculations) can be expressed as follows. If some forms $\xi^{1}, \ldots, \xi^{n}$ provide a basis of $\Phi / \Omega$ then, for a fixed $\omega \in \Omega$, $\omega \notin \Omega^{\prime}$ we have $\mathrm{d} \omega=\sum k_{i} \xi^{i} \wedge \omega$ modulo $\Omega \wedge \Omega$ and $\Omega^{\prime}$. We search for nonvanishing $f \in \mathscr{F}$ which satisfy $\mathrm{d}(\omega / f)=0$ modulo $\Omega \wedge \Omega$ and $\Omega^{\prime}$. Assuming $\mathrm{d} f=\sum f_{j} \xi^{j}$ modulo $\Omega$, we obtain the conditions

$$
f_{i}-f k_{i}=0 \quad(i=1, \ldots, n)
$$

equivalent to the above (and in reality identical if the bases are chosen dual, $\xi^{i}\left(Z_{j}\right) \equiv$ $\equiv \delta_{j}^{i}$, since then $f_{i} \equiv Z_{i} f$ and $k_{i} \equiv h_{i}$ ). 
21. Simple examples. (i) We begin with the module of $n$-dimensional contact forms. To this aim, let the coordinates in $\mathbb{R}^{\infty}$ be denoted (a little artifically) by

$$
\begin{aligned}
& x^{i}, y_{I}^{j} \quad\left(i=1, \ldots, n ; j=1, \ldots, m ; I=i_{1} \ldots i_{k}\right. \\
& \text { with } \left.k=0,1, \ldots \text { and } i_{1}, i_{2}, \ldots=1, \ldots, n\right)
\end{aligned}
$$

where the order of the entries $i_{1}, \ldots, i_{k}$ of the multiindex $I$ is inessential (so that $I$ can be arranged in the non-decreasing order $i_{1} \leqq \ldots \leqq i_{k}$ if uniqueness of the record is needed). Then the module $\Omega=\Omega(n, m)$ of contact forms is generated by all $\omega_{I}^{j}=\mathrm{d} y_{I}^{j}-\sum y_{I i}^{j} \mathrm{~d} x^{i}$ and $\mathscr{H}=\Omega^{\perp}$ by the familiar total derivatives $\partial_{i}=\partial / \partial x^{i}+$ $+\sum^{\infty} y_{I i}^{j} \partial / \partial y_{I}^{j}$. The formulae $\mathrm{d} \omega_{I}^{j} \equiv \sum \mathrm{d} x^{i} \wedge \omega_{I i}^{j}, \mathscr{L}_{\partial_{i}} \omega_{I}^{j} \equiv \omega_{I i}^{j}$ easily imply that $\Omega$ is a diffiety. The generalized symmetries $S \in \Phi^{\wedge}$ of $\Omega$ can be easily calculated if we use the basis of $\Phi$ consisting of all forms $\mathrm{d} x^{i}, \omega_{I}^{j}$ and the dual weak basis of $\Phi^{\wedge}$ consisting of all vector fields $\partial_{i}, \partial / \partial \omega_{I}^{j}$. Assuming $S=\sum s_{i} \partial_{i}+\sum s_{I}^{j} \partial / \partial \omega_{I}^{j}$, we can make the conditions $\mathscr{L}_{S} \Omega \subset \Omega$ more explicit by writing them in the form

$$
\left.\left.\mathscr{L}_{S} \omega_{I}^{j} \equiv(\mathrm{d} S\urcorner+S\right\urcorner \mathrm{~d}\right) \omega_{I}^{j}=\mathrm{d} s_{I}^{j}+\sum s_{i} \omega_{I i}^{j}-\sum s_{I i}^{j} \mathrm{~d} x^{i} \in \Omega
$$

so that the functions $s_{i}, s^{j} \in \mathscr{F}(I=\emptyset$ is empty here) may be chosen quite arbitrarily and the remaining coefficients $s_{I}^{j}(I=\emptyset)$ are recurrently determined by the rule $s_{I i}^{j} \equiv \partial_{i} s^{j}$, that is $s_{I}^{j}=\partial_{I} s^{j}$.

(ii) Continuing (i), let us assume $m=1$. If $S$ is a generalized symmetry, then $(*)$ reduces to

$$
\mathscr{L}_{S} \omega_{I}^{1} \equiv \sum a_{I I^{\prime}} \omega_{I^{\prime}}^{1}+\sum s_{i} \omega_{I i}^{1} \quad\left(a_{I I^{\prime}} \equiv \partial s_{I}^{1} / \partial y_{I^{\prime}}^{1}=\partial \partial_{I^{\prime}} s^{1} / \partial y_{I^{\prime}}^{1}\right) .
$$

In particular, $\mathscr{L}_{S} \omega^{1}=\sum \partial s^{1} / \partial y_{I^{\prime}}^{1} \cdot \omega_{I^{\prime}}^{1}+\sum s_{i} \omega_{I i}^{1}$. If some contact form $\omega_{I^{\prime \prime}}^{1}\left(I^{\prime \prime}=\right.$ $=i_{1}^{\prime \prime}, \ldots, i_{k}^{\prime \prime}$ ) with $\left|I^{\prime \prime}\right|=k>1$ effectively appears on the right hand side of the latter formula, then one can easily observe that the right hand side of $(* *)$ contains a nontrivial summand with $\omega_{I^{\prime \prime}}^{1},\left|I^{\prime \prime}\right|=k+|I|$ and hence $S \notin \boldsymbol{G}$ (cf. point (iv) of Section 10). So the inclusion $S \in \boldsymbol{G}$ implies $\mathscr{L}_{S} \omega^{1}=h \omega^{1}$ (with an appropriate $h \in \mathscr{F}$ ) or, in classical terms, $S$ is an infinitesimal contact transformation. Since the converse implication is well-known, we have obtained a result which is an invariant reformulation of the Bäcklund theorem concerning the non-existence of "higher order contact symmetries". (Note aside that the original definition of these symmetries consisting in the property that they preserve the submodule generated by contact forms $\omega_{I}^{j}$ with $|I| \leqq$ some constant makes little sense from our point of view.)

(iii) Continuing (ii), let us consider the overdiffieties $\Omega^{\prime}$ of $\Omega$ with $\ell\left(\Omega / \Omega^{\prime}\right)=1$. Clearly $\omega^{1} \notin \Omega^{\prime}$ (in the other case all $\mathscr{L}_{\mathscr{H}}^{k} \omega^{1} \subset \Omega^{\prime}$, hence $\Omega \subset \Omega^{\prime}$ ) so that $\Omega^{\prime}$ is generated by certain forms $\omega_{I}^{1}-g_{I} \omega^{1}\left(I \neq \emptyset, g_{I} \in \mathscr{F}\right)$. Since

$$
\mathscr{L}_{\partial_{i}}\left(\omega_{I}^{1}-g \omega^{1}\right)=\omega_{I i}^{1}-\partial_{i} g_{I} \cdot \omega^{1}-g_{I} \omega_{i}^{1} \in \Omega^{\prime}
$$

implies $\omega_{I i}^{1}-\left(\partial_{i} g_{I}+g_{I} g_{i}\right) \omega^{1} \in \Omega^{\prime}$ we obtain the recurrence $g_{I i} \equiv \partial_{i} g_{I}+g_{I} g_{i}$ for the unknown coefficients $g_{I}$. One can verify that this recurrence ensures the flatness of $\Omega^{\prime}$, too, hence $\Omega^{\prime}$ is indeed an overdiffiety of $\Omega$. According to Section 20 (with 
$\xi^{i} \equiv \mathrm{d} x^{i}, Z_{i} \equiv \partial_{i}, h_{i} \equiv g_{i}$ ), the overdiffiety $\Omega^{\prime}$ arises from a generalized symmetry $S=f Y$ of $\Omega$ if and only if the system $\partial_{i} f \equiv f g_{i}$ has a solution $f \in \mathscr{F}$. For an arbitrary choice of $g_{1}, \ldots, g_{n}$, this is clearly contradictory. However, one may choose $g_{i} \equiv$ $\equiv \partial_{i} s^{1} / s^{1}$ and then $S=\sum^{\infty} s_{I}^{1} \partial / \partial \omega_{I}^{j}$ appears just the same as in (i). It follows that other solutions do not exist.

(iv) Continuing (i) with $n=m=1$, we shall abbreviate $x^{1}=x$ and $y_{I}^{1} \equiv y_{k}$, $\omega_{I}^{1} \equiv \omega_{k}, s_{I}^{1} \equiv s_{k}$ whenever $|I|=|1 \ldots 1|=k$ ( $k$ terms). So the coordinates are $x, y_{0}, y_{1}, \ldots$, the module $\Omega=\Omega(1,1)$ is generated by the contact forms $\omega_{k}=\mathrm{d} y_{k}-$ $-y_{k+1} \mathrm{~d} x$ and $\mathscr{H}=\Omega^{\perp}$ consists of all multiples of the vector field $\partial=\partial_{1}=$ $=\partial / \partial x+\sum^{\infty} y_{k+1} \partial / \partial y_{k}$. For a generalized symmetry $S=s \partial+\sum^{\infty} s_{k} \partial / \partial \omega_{k} \quad$ (where $\left.s_{k} \equiv \partial^{k} s_{0}\right)$, the corresponding evolution diffiety $\bar{\Omega}$ is generated by the forms

$$
\bar{\omega}_{k}=\omega_{k}-s_{k} \mathrm{~d} t=\mathrm{d} y_{k}-y_{k+1} \mathrm{~d} x-s_{k} \mathrm{~d} t \quad(k=0,1, \ldots),
$$

where $t=t^{0}$ is the additional variable. The module $\mathscr{H}=\bar{\Omega}^{\perp}$ is generated by two commuting vector fields $\partial$ (considered in the extended space) and $\partial^{\prime}=(\partial / \partial t)+$ $+\sum^{\infty} s_{k} \partial / \partial y_{k}$. A vector field $\bar{S}=s^{\prime} \partial^{\prime}+\bar{s} \partial+\sum^{\infty} \bar{s}_{k} \partial / \partial \bar{\omega}_{k}$ is a generalized symmetry of $\bar{\Omega}$ if $\mathscr{L}_{\bar{S}} \bar{\Omega} \subset \bar{\Omega}$ which yields the requirements

$$
\bar{s}_{k+1} \equiv \partial \bar{s}_{k}, \quad \partial^{\prime} \bar{s}_{l} \equiv \sum \partial s_{l} l \partial y_{k} \bar{s}_{k} \quad(k, l=0,1, \ldots) .
$$

The first group is a simple recurrence and it may be shown that already the initial equation $(l=0)$ of the second group expressed in terms of $\bar{s}_{0}$ (i.e., $\partial^{\prime} \bar{s}_{0}=$ $\left.=\sum\left(\partial s_{0} / \partial y_{k}\right) \partial^{k} s_{0}\right)$ is sufficient. (In fact, the recurrence ensures the commutation $[\partial, S]=(\partial s / \partial x) \partial$ and owing to it, the initial equation $l=0$ (which is equivalent to $\left.\mathscr{L}_{\bar{S}} \bar{\omega}_{0} \subset \bar{\Omega}\right)$ implies

$$
\mathscr{L}_{\bar{S}} \omega_{1}=\mathscr{L}_{\bar{S}} \mathscr{L}_{\partial} \bar{\omega}_{0}=\mathscr{L}_{\partial} \mathscr{L}_{\dot{\bar{S}}} \bar{\omega}_{0}-\mathscr{L}_{[0, \bar{S}]} \bar{\omega}_{0} \subset \bar{\Omega}
$$

and analogously $\mathscr{L}_{\bar{S}} \bar{\omega}_{k} \subset \bar{\Omega}(k=2,3, \ldots)$, that is $\mathscr{L}_{\bar{S}} \bar{\Omega} \subset \bar{\Omega}$.) Let us mention the overdiffieties $\bar{\Omega}^{\prime}$ of $\bar{\Omega}$ with $\ell\left(\bar{\Omega} / \bar{\Omega}^{\prime}\right)=1$. They are generated by certain forms $\bar{\omega}_{k}-g_{k} \bar{\omega}_{0}(k=1,2, \ldots)$ and one can obtain the requirements

$$
\begin{aligned}
& g_{k+1}=\partial g_{k}+g_{k} g_{1}, \quad \sum g_{k} \partial \bar{s}_{l} / \partial y_{k}=g_{l} \sum g_{k} \partial \bar{s}_{0} / \partial y_{k}+\partial^{\prime} g_{l} \\
& (k, l=1,2, \ldots) .
\end{aligned}
$$

In reality, only the initial equation $l=1$ of the second group is important.

(v) We pass to a generalization of the Bäcklund theorem. We shall deal with a system of $m$ ordinary differential equations involving $m+1$ unknown functions. In term of diffieties, we deal with a diffiety $\Omega$ satisfying $\ell(\mathscr{H})=\mu(\Omega)=1$. After some rearrangements, the diffiety is generated by the forms

$$
\begin{aligned}
& \omega_{k}=\mathrm{d} y_{k}-y_{k+1} \mathrm{~d} x(k=0,1, \ldots), \\
& \vartheta^{i}=\mathrm{d} z^{i}-f^{i}\left(x, z^{1}, \ldots, z^{m}, y_{0}, y_{1}\right) \mathrm{d} x \quad(i=1, \ldots, m),
\end{aligned}
$$

where $x, z^{1}, \ldots, z^{m}, y_{0}, y_{1}, \ldots$ are coordinates in the underlying space and $f^{1}, \ldots, f^{m} \in$ 
$\in \mathscr{F}$ are given functions. (The diffiety corresponds to the Monge system $\mathrm{d} z^{i} / \mathrm{d} t \equiv$ $\equiv f^{i}\left(x, z^{1}, \ldots, z^{m}, y, \mathrm{~d} y / \mathrm{d} x\right)$.) One can see that $\mathscr{H}=\Omega^{\perp}$ consists of all multiples of the vector field $Z=\partial / \partial x+\sum f^{i} \partial / \partial z^{i}+\sum^{\infty} y_{k+1} \partial / \partial y_{k}$. If the basis of $\Phi$ consisting of all forms $\mathrm{d} x, \vartheta^{i}, \omega_{k}$ and the relevant weak dual basis of $\Phi^{\wedge}$ are used, the conditions for the coefficients of a generalized symmetry $S=s Z+\sum r^{i} \partial / \partial \vartheta^{i}+$ $+\sum^{\infty} s_{k} \partial / \partial \omega_{k}$ are as follows:

$$
\begin{aligned}
& s_{k+1}=Z s_{k}(k=0,1, \ldots), Z r^{i}=\frac{\partial f^{i}}{\partial y_{0}} s_{0}+\frac{\partial f^{i}}{\partial y_{1}} s_{1}+\sum \frac{\partial f^{i}}{\partial z^{j}} r^{j} \\
& (i=1, \ldots, m) .
\end{aligned}
$$

The first group of conditions is a simple recurrence, the second group consists of $m$ equations for $m+1$ unknown functions $r^{1}, \ldots, r^{m}, s_{0}\left(\right.$ with $\left.s_{1}=Z s_{0}\right)$ and may be investigated quite separately. Alas, this is a difficult problem and we restrict ourselves to the following result: every generalized symmetry $S \in \boldsymbol{G}$ is an infinitesimal contact transformation modulo $\vartheta^{1}, \ldots, \vartheta^{m}$, that is $\mathscr{L}_{S} \omega_{0}=h \omega_{0}+\sum h_{i} \vartheta^{i}$. (Proof. Assume the formula $\mathscr{L}_{S} \omega_{0}=(\ldots)+A \omega_{M}$ with $A \neq 0, M>1$ holds, where (..) denotes some summands involving $\vartheta^{1}, \ldots, \vartheta^{m}$ and the lower order $\omega_{k}$ (with $k<M$ ). Owing to $\mathscr{L}_{Z} \vartheta^{i}=(\ldots)+B_{i} \omega_{1}, \mathscr{L}_{\mathrm{Z}} \omega_{k}=\omega_{k+1}$ and $[S, Z] \in \mathscr{H}$, we conclude

$$
\begin{aligned}
& \mathscr{L}_{S} \omega_{k+1}=\mathscr{L}_{S} \mathscr{L}_{\mathrm{Z}} \omega_{k}=\mathscr{L}_{\mathrm{Z}} \mathscr{L}_{S} \omega_{k}+\mathscr{L}_{[S, Z]} \omega_{k}=(\ldots)+A \omega_{M+k+1} \\
& (k=0,1, \ldots)
\end{aligned}
$$

by induction. Let analogously $\mathscr{L}_{S} \vartheta^{i}=(\ldots)+C_{i} \omega_{N}{ }_{i}{ }^{\prime}$. Then $\mathscr{L}_{S}^{k} \vartheta^{i}=(\ldots)+$ $+C_{i} A^{k} \omega_{N(i)+k M}$ so that $S \notin \boldsymbol{G}$ according to (iv), Section 10.)

(vi) In terms of the coordinates $x, y_{0}, z_{0}, y_{1}, z_{1}, \ldots$, let $\Omega=\Omega(1,2)$ be the diffiety generated by all forms $\omega_{k}=\mathrm{d} y_{k}-y_{k+1} \mathrm{~d} x, \vartheta_{k}=\mathrm{d} z_{k}-z_{k+1} \mathrm{~d} x(k=0,1, \ldots)$. Then $S=\sum z_{c+k} \partial / \partial \omega_{k}$ is a generalized symmetry and $\mathscr{L}_{S} \omega_{k} \equiv \vartheta_{c+k}, \mathscr{L}_{S} \vartheta_{k} \equiv 0$ so that $S \in \boldsymbol{G}$. Here $c=0,1, \ldots$ is a fixed constant. The corresponding group $F_{\lambda}$ which preserves $\Omega$ is given by $F_{\lambda}^{*} y_{k} \equiv y_{k}+\eta z_{c+k}, F_{\lambda}^{*} z_{k} \equiv z_{k}, F_{\lambda}^{*} x=x$. The example can be easily generalized and it follows that the modules $\Omega(n, m)$ of contact forms admit huge families of automorphisms not preserving the submodules generated by the forms $\omega_{I}^{j}$ with $|I| \leqq$ constant. It follows that the common theory of partial differential equations based on the fixed hierarchy of dependent and independent variables is (a little) misleading.

22. Concluding remarks. Infinitesimal transformations (vector fields) lying in $\boldsymbol{G}$ should undertake the role of the ambigous "classical infinitesimal transformations" which are defined from case to case by the property of preserving a certain fixed space of variables. It is to be noted that all infinitesimal transformations $Z$ of a LieCartan pseudogroup (defined by the property $\mathscr{L}_{\mathrm{Z}} \mathscr{K}=\mathscr{L}_{\mathrm{Z}} \Xi=0$ where $\mathscr{K}, \Xi$ are the invariants and Maurer-Cartan forms, respectively) are lying in $\boldsymbol{G}$ as follows from (v) Section 10. On the contrary, infinitesimal automorphisms $S$ of such 
a pseudogroup (defined by $\mathscr{L}_{S} \mathscr{K} \subset \mathscr{K}, \mathscr{L}_{S} \Xi \subset \Xi$ ) need not apriori belong to $\boldsymbol{G}$ except, e.g., the special case when the Maurer-Cartan forms admit a canonical filtration (preserved by all automorphisms) by finitely generated submodules. (All simple transitive pseudogroups are of this kind.) We do not know whether all infinitesimal automorphisms lying in $\boldsymbol{G}$ of a geometrical object constitute an infinitesimal pseudogroup.

The problem of determination of all generalized symmetries of a diffiety may be reduced to a finite number of recurrences and a separate (linear in derivatives) system of equations involving more unknown functions than equations (cf. the particular examples in Section 21); in general the proof is based on the concept of involutiveness. In certain favourable cases, it may be reduced to the classical problem of equivalence. (The point (v) of Section 21 seems to be instructive. One can observe that every submodule generated by the forms $\vartheta^{1}, \ldots, \vartheta^{m}, \omega_{0}, \ldots, \omega_{N}$ where $N \geqq N(i), i=1, \ldots, m$, is preserved by $\mathscr{L}_{S}$. Then the constant $N$ can be uniformly reduced to $N=1$ by using the derived submodules which are preserved, too.) Quite analogous remarks can be repeated for overdiffieties.

Note aside that the evolution equations admitting an infinite number of generalized symmetries (the integrable equations) belong to the most interesting topics of today's mathematics.

As we have seen in Section 12, nonvanishing vector fields from $\boldsymbol{G}$ are essentially the same as the vector field $\partial / \partial t^{1}$. On the contrary, the family $\boldsymbol{F}$ includes quite different objects (cf., e.g., the invariant $\mu(\Omega)$ ) and the problem of classification proves to be extremely difficult (but highly important for the theory of the Monge systems). Also the weaker (and rather natural) concept of projectable vector fields from $\boldsymbol{F}$ is very interesting in connection with certain classical problems, and far from being trivial.

In more detail, let $Z \in \boldsymbol{F} \subset \Phi^{\wedge}(\boldsymbol{U}), \bar{Z} \in \bar{F}=\boldsymbol{F}(\bar{U}) \subset \Phi^{\wedge}(\bar{U})$ be given vector fields. If $F: U \rightarrow \bar{U}$ is a surjection and $Z F^{*} f=\bar{Z} f$ for every $f \in \mathscr{F}(\bar{U}), Z$ and $\bar{Z}$ are said to be $F$-related (and $Z$ is projectable into $\bar{Z}$ ). In terms of differential forms, $\bar{\Omega}=\bar{Z}^{\perp}$ is called a factordiffiety of $\Omega=Z^{\perp}$ (and $\Omega$ is a covering of $\bar{\Omega}$ ); we suppose $Z \neq 0$, $\bar{Z} \neq 0$ here. If $\bar{\Omega}$ is another factordiffiety of $\Omega$, then $\bar{\Omega}$ and $\bar{\Omega}$ are said to be in Bäcklund correspondence. Several authors propose to use this term only when the fibers of the relevant surjections are of finite dimensions. Then many properties of $\bar{\Omega}, \bar{\Omega}$ are the same (e.g. $\mu(\bar{\Omega})=\mu(\bar{\Omega}))$. The definitions can be carried over to general diffieties $\ell(\mathscr{H})>1$ without much effort.

23. Added in proof. If $F_{\lambda}$ is a group (cf. section 5) then all pull-backs $F_{\lambda}^{*} t^{i}(i$ is fixed but $\lambda$ ranges in $\mathbb{R}$ ) can be expressed by a finite number of coordinates (namely by $\left.t^{1}, \ldots, t^{m(i)}\right)$ so that there exists a finite family $h^{1}=t^{i}, h^{2}, \ldots, h^{n(i)} \in \mathscr{F}$ such that $F_{\lambda}^{*} h^{j}=H^{j}\left(h^{1}, \ldots, h^{n(j)}\right)$ is a composed function (use the group property). It follows that (under certain regularity assumptions) the coordinates $t^{1}, t^{2}, \ldots$ can be changed in such a manner that $F_{\lambda}^{*}$ preserves every space of variables $t^{1}, \ldots, t^{n(i)}(i=1,2, \ldots)$ 
where $n(i) \rightarrow \infty$ as $i \rightarrow \infty$. In brief terms, $F_{\lambda}^{*}$ preserves an appropriate finitedimensional filtration of the variables. Such a filtration need not exist for a general automorphism $F$ of the space $\mathbb{R}^{\infty}$ in the sense of section 4 , of course. But at the same time, this $F$ may preserve a quite nice geometrical object (cf. the example below). It follows that automorphisms of a geometrical object need not constitute the LieCartan pseudogroup (cf section 22) so that they cannot be universally calculated by the Cartan's moving frames! (Example. Consider the object $\Omega(1,2)$ of $(1)$ section 21. Using a simplified notation, this is the module generated by the contact forms $\vartheta_{i}=\mathrm{d} y_{i}-y_{i+1} \mathrm{~d} x, \omega_{i}=\mathrm{d} z_{i}-z_{i+1} \mathrm{~d} x(i=0,1, \ldots)$ in the space of the variables $x, y_{0}, z_{0}, y_{1}, z_{1}, \ldots$. Then the groups $F_{\lambda}, G_{\lambda}$ defined by $F_{\lambda}^{*} x=G_{\lambda}^{*} x=x, F_{\lambda}^{*} y_{i}=y_{i}$ $F_{\lambda}^{*} z_{i}=z_{i}+\lambda y_{i+1}, G_{\lambda}^{*} y_{i}=y_{i}+\lambda z_{i+1}, G_{\lambda}^{*} z_{i}=z_{i}$ preserve $\Omega(1,2)$ so that $H_{\lambda}=$ $=F_{\lambda} \circ G_{\lambda}$ is an automorphism, too. But $H_{\lambda}^{*} y_{i}=y_{i}+\lambda z_{i+1}+\lambda^{2}\left(z_{i+1}+y_{i+2}+\right.$ $\left.+z_{i+3}\right)$ thus $H_{\lambda}^{*}$ does not preserve any filtration of the above kind.)

\section{Bibliography}

[1] R. L. Anderson, N. H. Ibragimov: Lie-Bäcklund Transformations in Applications, SIAM Philadelphia 1979.

[2] M. J. Ablowitz, M. Segur: Solitons and the Inverse Scattering Transform, SIAM Philadelphia 1981 (Russian transl. Moskva 1987).

[3] J. Chrastina: On formal theory of differential equations, Časopis pro pěst. matem. Praha; Part I, vol. 111 (1986), 353-383; Part II, vol. 114 (1989), 60-105; Part III (to appear).

[4] J. Chrastina: Formal Calculus of Variations on Fibered Manifolds, Folia UJEP Brno 1989, Mathematica 2.

[5] N. H. Ibragimov: Transformation Groups in Mathematical Physics (Russian), Moskva, Nauka 1983.

[6] Ju. I. Manin: Algebraic aspects of nonlinear differential equations (Russian), Itogi Nauki i Techniki VINITI Ser. Sovrem. Problemy matematiki 11 (1978), 5-152.

[7] P. J. Olver: Applications of Groups to Differential Equations, Springer-Verlag 1986.

[8] V. V. Sokolov: On the symmetries of evolutional equations (Russian), Uspěchi matem. nauk 43 , vyp. 5 (263) $1988,133-163$.

Author's address: 66295 Brno, Janáčkovo nám. 2a, Czechoslovakia (katedra matematiky UJEP). 